\section{Parametric adjustment to a shifting target alternating with saccades to a stationary reference point}

\author{
GLENN E. WEISFELD \\ Tufts University, Medford, Mass. 02155
}

The oculomotor system can progressively improve the accuracy of repeated saccades to a target that shifts in midsaccade, by utilizing retinal feedback to diminish the induced error. The present study investigated whether this parametric adjustment compromises S's ability to fixate the stationary reference point on alternating saccades to the other side of his visual field. Fixation of the reference point on shifting-target runs was sometimes even more accurate than fixation on stationary-target runs. The capacity of the narametric adjustment mechanism to adapt to specific targets was thereby demonstrated.

The role of the central nervous system in visual perception is being elucidated very rapidly. It is easy to forget the essential role of the peripheral nervous system in relaying visual information to the CNS and the fact that the latter plays an active part in this process. The present article discusses the mechanism by which the oculomotor system improves the accuracy of its saccades, a mechanism which depends not only upon Cranial Nerves II, III, IV, and VI, but also, presumably, upon visual reflex centers and memory stores of the higher structures.

In 1901, Dodge and Cline established that a single saccade occurs too rapidly to utilize proprioceptive feedback from the extraocular muscles. When these muscles fail to contract precisely, as necessary to fixate a target, midcourse correction mediated by proprioceptive cues is impossible.

The oculomotor system can, and does, however, execute subsequent saccades to home in on the target. Because of the time lag between one saccade and the next, these remedial saccades hypothetically could benefit from proprioceptive feedback from the extraocular muscles (Ludvigh, 1952 ). Errors caused by muscle fatigue could be fed back, in the form of proprioceptive information, to be utilized in readjusting the oculomotor system for more accurate future saccades. If such a mechanism were operating, then constant errors of fixation could be corrected fundamentally rather than being left to the device of uninformed remedial saccades which would repeat the constant error, albeit on a reduced scale.

McLaughlin (1967) established the existence of such a corrective process; at the same time, he demonstrated that this mechanism cannot rely upon proprioceptive feedback.

McLaughlin investigated repeated saccades from the same reference point to the same target, back and forth, using $S$ 's right eye only. McLaughlin induced a constant error in fixation by constructing an apparatus that mechanically shifted the target slightly to one side during S's saccade toward the target. Each time that $S$ tried to fixate the target by making a single saccade from the reference point over to the target visibile in his peripheral field of vision, he would overshoot or undershoot the shifting target.

However, S progressively reduced these errors with each trial. Parametric adjustment took place: the oculomotor system was recalibrated so as to compensate for this constant error. The same corrective mechanism would be capable of remedying the constant errors caused by extraocular muscle fatigue and by other systematic flaws.

Furthermore, no proprioceptive feedback was involved in this parametric adjustment. The extraocular muscles presumably were midsaccadic shifting of the target induced an "error" artificially.

This error could be detected, however, by the retina, since a discrepancy in retinal distance would exist between the point of fixation (the fovea) and the position of the errant target. Apparently it is this information which the oculomotor system utilizes in performing parametric adjustment.

The hypothesis that retinal feedback, rather than proprioceptive feedback, mediates saccadic eye movements is well established (Robinson, 1968). For example, Fender \& Nye (1961) found that the magnitude of the normal flick, drift, and tremor deviations accompanying otherwise steady fixation of a target corresponded in magnitude to predictions based upon the known sensitivity of the retinal feedback contracting normally, but the system. But only with McLaughlin's work was the existence of parametric adjustment confirmed and its guidance by the same retinal feedback demonstrated. Proprioceptive cues are entirely irrelevant, then, to the initiation of saccades, to midcourse correction of saccades, and to parametric adjustment to constant errors over a series of saccades.

The capacities and limitations of the retinal feedback system in parametric adjustment, however, are still somewhat obscure. McLaughlin's experiment did not include an assessment of the accuracy with which the stationary reference point was fixated. Perhaps parametric adjustment to the shifting target on one side of the visual field detracted from the accuracy of fixating the reference point on the opposite side during alternate saccades.

The present investigation, then, repeated McLaughlin's basic trompe-l'oeil technique, but in a way designed to determine if the oculomotor system can perform both fixations accurately. In addition, the effect of side (nasal or temporal) on parametric adjustment was tested.

Thus, four conditions were included: (1) both the reference point and the target stationary, with the target (the object of the first saccade in series of left-right-left saccades) on the right (temporal side; right eye used exclusively); (2) the target shifting, reference point stationary as always, with the target on the right; (3) the target stationary, target on the left (nasal) side; and (4) the target shifting, on the left.

The effects of target side and of shifting on the accuracy of the return saccades to the stationary reference point were evaluated, using several planned comparisons: (A) Condition 1 vs Condition 2--effect of shifting on trials with the target on the left; (B) Condition 3 vs Condition 4-effect of shifting on trials with the target on the right; (C) Condition 1 vs Condition 3-effect of target side, without shifting; and (D) Condition 2 vs Condition 4-effect of target side, with shifting.

The Ss were three male Tufts University students who understood the experiment completely throughout their participation. No extrinsic incentives were employed. Ss' vision was emmetropic.

\section{APPARATUS}

The S's head was fixed with the aid of an individualized biteplate. The left eye was covered.

Eye position was monitored after the method of Torok, Guillemin, \& Barnothy (1951). A red light was reflected off S's eye. Part of the 
Table 1

Accuracy of Fixation in Minutes of Arc

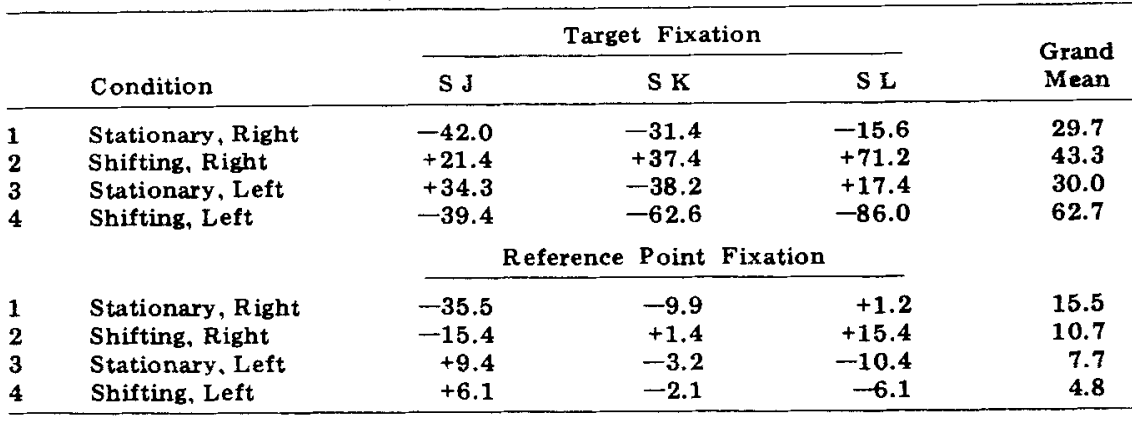

Note-Negative numbers refer to errors to the left; positive to the right. The grand means are derived from the absolute values of individual scores. Each individual score is the mean of 24 trials.

reflected light was admitted through a horizontal split in an opaque filter. The filter was adjusted so that the slit admitted light reflected off S's sclera and iris. A viewing screen constructed as an aid to adjusting the apparatus revealed the position of the slit relative to the iris and adjacent sclera.

When $\mathrm{S}$ rotated his eye from side to side, the stationary slit fell over varying amounts of sclera and iris. When the eye was moved to the extreme left, the slit admitted only light reflected from the white sclera. When the eye was moved to the extreme right, the slit admitted only light from the darker iris. Thus, the intensity of light reflected off the surface of the eye and through the slit varied with the position of the eye, i.e., with S's direction of gaze.

The intensity of this beam of light was amplified and measured by a photodiode. With the aid of a PDP-5 computer, eye position in degrees and minutes of arc was monitored with suitable precision and reliability.

The targets and reference points were five vertical white lights stationed side by side in a plane perpendicular to S's line of sight and at a distance of $147.6 \mathrm{~cm}$ from his eye. The distance between the extreme left and the extreme right lights was $26.6 \mathrm{~cm}$, so that the angle subtended by these two lights was $10 \mathrm{deg} 0 \mathrm{~min}$. The distances between the five lights were, respectively, 2, 3, 3, and 2 deg. Each light measured $30.5 \mathrm{~cm}$ in height. Half-way down each light, black cross hairs designated the precise $0.4-\mathrm{cm}-$ diam area of the light to be fixated.

\section{PROCEDURE}

For each run of 18 initial and 18 return fixations, the slit was adjusted with respect to the sclera and iris, for three eye positions, which corresponded to fixation of three lights (far left, center, and far right).

After this alignment, the room was darkened and $S$ pressed a button to begin the further presentation of lights. These next lights permitted calibration of the eye-position detector. $\mathrm{S}$ was instructed to fixate the cross hairs of each of the five lights as it appeared and, when satisfied that he was fixating the light accurately, to press the button. This triggered a sampling of eye position and then replaced the last light with the next one. S was instructed to blink until comfortable, fixate the new light, and press the button. This was repeated for each of the five lights, twice through the sequence.

When the calibration was completed, $\mathrm{S}$ was to abandon the button and await presentation of the lights of the experimental run. First, the center light went on as a warning, and then one of the two end lights appeared singly, depending on whether the run in point began on the left (Conditions 1 and 2) or the right (Conditions 3 and 4). S was instructed to fixate that reference-point light and to continue to do so even after the target light appeared along with it. Only when a buzzer sounded was $S$ to move his gaze over to the target light, visible in his peripheral field of vision. After moving his gaze from the reference-point light to the target light, $S$ was to keep his gaze fixated upon the target light until it went off, at which time he was to move his gaze back to the reference-point light, which had remained on all the time. S then was to blink a few times and await the next trial, consisting of an initial movement from the reference point to the target and a return movement to the reference point, as before.

During the initial movement toward the target, the passing of the eye position, as detected by the apparatus, across the midpoint of the range triggered a timing device which both turned off the buzzer and ordered a sample of eye position to be taken a few milliseconds thereafter, when the saccade would be completed but before a second saccade could be initiated. Similarly, on the return eye movement to the reference point, the timer took a reading at the appropriate instant. The subsequent trials were initiated by the computer program independently of S's eye movements.

The first reference-point light to appear in a trial was, as stated previously, either the extreme left or the extreme right light. The second target light was the second-from-theright or second-from-the-left light, on the side opposite from the first. For shifted-target trials, however, the extreme left or extreme right light came on briefly instead of the second-from-the-left or -right light, before being replaced by the latter. This replacement or "shifting" was triggered by the passing of the line of gaze over the midpoint of the range, so that the saccade was already under way and could not be corrected in response to the shift of target. This shifting, in runs when it occurred, appeared only in the initial target-fixating half of Trials 8-13. For this reason, only the data for Trials 8-13 were used, for both shifting and stationary runs.

For each $S$ on each of 4 days, four runs of 18 saccades in each direction, initial and return, were conducted. Each of the four runs involved a different condition, and the sequential order of presentation of conditions was varied randomly. Thus, for each $\mathrm{S}$, each condition occurred once in each of the four serial positions for each of the 4 days: a 4 by 4 Latin-square design.

\section{RESULTS}

It was first determined if parametric adjustment to shifting targets had, in fact, occurred. Table 1 shows that the accuracy for shifting-target runs, Conditions 2 and 4 , was appreciable, considering that $\mathbf{S}$ initiated his saccade with the preshift, decoy target a full $120 \mathrm{~min}$ away from the postshift, true target. These results confirmed the presence of parametric adjustment. Errors, as expected, were in the direction of the decoy lights. Fixation of stationary targets, Conditions 1 and 3 , was somewhat more accurate than fixation of shifting targets, also as expected.

The return saccades to the stationary reference points were also highly accurate, even for runs with shifting targets on the initial saccades, Conditions 2 and 4 . Fixation of reference points was even more accurate than fixation of targets for all four conditions. This suggests that $S$ relied on the reference point the initial point of fixation for a given experimental run-as an anchor point in his visual field, regardless of whether the target was shifting or stationary. 
Analyses of variance were carried out for each $\mathrm{S}$ to determine the effects of condition, day, and serial position within a day on the accuracy of return saccades to the reference point. For none of the Ss was the effect of serial position significant. Only for $\mathrm{S} \mathrm{K}$ was the effect of day significant $(F=10.4$, $\mathrm{df}=3 / 15, \mathrm{p}<.01$ ). This was probably a practice effect.

Significant differences did exist, however, across conditions for two Ss $\mathrm{J}(\mathrm{F}=6.7, \mathrm{df}=3 / 15, \mathrm{p}<.05)$ and $\mathrm{K}$ $(\mathrm{F}=18.9, \mathrm{df}=3 / 15, \mathrm{p}<.01)$. Dunn's multiple comparison procedure revealed the source of these differences. For J, Comparisons A (20.1), C (26.1), and D (9.3) were significant $[\mathrm{d}=8.9, \quad \mathrm{~d} f=3 / 89$ $\operatorname{MS}($ error $)=89.2, a<.01]$. That is, the differences between the means of the two conditions in a given comparison exceeded the value of $D$ for these three comparisons. For $\mathrm{K}$, Comparisons $\mathrm{A}(8.5)$ and $\mathrm{C}(6.7)$ were significant $[\mathrm{d}=4.0, \quad \mathrm{df}=3 / 89$ $\operatorname{MS}($ error $)=18.3, \alpha<.01]$. None of the other comparisons for the three Ss reached significance at the .05 level.

Thus, two of the three Ss fixated the reference point even more accurately when the target shifted than when it was stationary, with the target on the right. This implies that parametric adjustment actually facilitated fixation of the reference point. No such effect was noted with the target on the left, however.

For the same two Ss, fixation of the reference point was more accurate when it was on the temporal side than on the nasal side. Only one of these two Ss manifested this difference when shifting was involved, however.

\section{DISCUSSION}

The $S$ was able to fixate both the target and the reference point accurately under all four conditions. Parametric adjustment to shifting targets did not detract from the accuracy of fixating the stationary reference points for any of the Ss. In fact, two of the three $S$ s fixated the reference points more accurately under one of the two shifting-target conditions than under the corresponding stationary-target condition.

Thus, the oculomotor system was able to perform two independent fixation tasks alternately, one of which involved parametric adjustment to a shifting target. The artificially induced constant error in fixating the shifting target was progressively diminished, while fixation of the stationary target on alternating saccades remained faithful.

Concerning the effect of side, the overall indifference of parametric adjustment to side further underlined the generality of the mechanism. Parametric adjustment operated satisfactorily to either side, although in half the comparisons, fixation of reference points on the temporal side was significantly more accurate than on the nasal side.

Parametric adjustment thus exhibits some resilience and adaptability, the extent of which can be revealed only by future investigations.

These results might be compared with those obtained by Steinberg (1970) in a similar study. Steinberg measured the accuracy with which $S$ s fixated a target and a reference point, but in this case, both were shifting. Parametric adjustment to both was observed, but the target and the reference point shifted by the same amount in the same direction. Thus, Steinberg did not demonstrate that parametric adjustment can be performed while the oculomotor system retains its ability to fixate a stationary reference point.

The present study, however, established that parametric adjustment is not merely a way for the oculomotor system to diminish constant errors caused by muscle fatigue and other such general aberrations. More than that, parametric adjustment allows the eye to improve its performance of specific, independent fixation tasks.

For example, consider a baseball batter following the flight of the ball. Although the ball is thrown too rapidly to be tracked by pursuit movements, apparently the batter keeps it in view by a series of saccades. If the batter improves his batting performance over time, perhaps he is benefiting from parametric adjustment to this particular target. That is, his extraocular muscles are not failing him-no general correction is required but a specific parametric adjustment is called for. The ability of the oculomotor system to perform these specific adjustments may be responsible for a batter's ability to learn to hit certain pitches without sacrificing his competence at such other visual-motor tasks as fielding batted balls.
Therefore, improvement at visual-motor tasks may not be merely a matter of refining the motor response required by a given visual perception, but may also involve improving perception-fixation-of the target. Parametric adjustment could be a key mechanism allowing the eye to improve at following targets which either appear too abruptly or move too rapidly for effective pursuit movements but which repeatedly travel at approximately the same velocity. Examples of such targets in man's evolutionary experience are fish or small mammals which appear suddenly and must be speared or otherwise struck quickly. The selective advantage afforded by the ability to perform parametric adjustments appropriate to specific targets thus becomes apparent.

It might be noted that in the present experiment, as in previous studies (e.g., McLaughlin, Kelly, Anderson, \& Wenz, 1968), Ss often performed parametric adjustments without being consciously aware that the target was shifting. This observation suggests that the dichotomies of voluntary vs reflexive responses, and operant vs classical conditioning, are simplifications which somewhat neglect the versatility and complexity of the mammalian nervous system.

\section{REFERENCES}

DODGE, R., \& CLINE, R. The angle velocity of eye movements. Psychological Review, 1901, 8, 145-157.

FENDER, D. H.. \& NYE, P. W. An investigation of the mechanisms of eye movement control. Kybernetik, 1961, 1 . $81-88$

LUDVIGH, E, Control of ocular movements and visual interpretation of the environment. Archives of Ophthalmology, 1952, 48, 442-448.

McLAUGHLIN. S. C. Parametric adjustment in saccadic eye movements. Perception \& Psychophysics, 1967, 2, 359-362.

MeLAUGHLIN, S. C.. KELLY, M. J., ANDERSON, R. E., \& WENZ. T. G. Localization of a peripheral target during parametric adjustment of saccadic eye movements. Perception \& Psychophysics, $1968,4,45-48$

ROBINSON. D. A. Eye movement control in primates. Science, 1968. 161. 1219-1224.

STEINBERG. B. D. Parametric adjustment of saccadic eye movements following induced errors in fixation. Paper read before the Optical Society of America. Miami, Fla., December 1970.

TOROK, N., GUILLEMIN, V., \& BARNOTHY, M. M. Photoelectric nystagmography. Annals of Otology, Rhinology, \& Laryngology, 1951. 60, 917-926. 